\title{
A simplified procedure for the study of memory retrieval processes*
}

\author{
LEE D. ROTHSTEIN $\dagger$ \\ Stanford University, Stanford, California 94305
}

\begin{abstract}
A simplified and inexpensive procedure for the conduct of research on recognition memory retrieval is presented. The procedure is a card sorting analog to Sternberg's item recognition task. An experiment is presented which demonstrates the card sorting task's validity. Discussion centers on the suitability of the task for the undergraduate laboratory. Suggestions are given for how card sorting may be used to study psycholinguistic processing and semantic memory.
\end{abstract}

Human information processing is an area of psychology that has shown enormous growth within the last 10 years. Unfortunately, this growth has not manifest itself in terms of widespread introduction into the undergraduate experimental psychology laboratory. Some of the reasons for this state of affairs have to do with the expense of such laboratory equipment as tachistoscopes, timers, stimulus displays, and stimulus sequencers, as well as the cost of computer time in analyzing such dependent variables as reaction time (RT).

One paradigm which seems ideally suited to the undergraduate laboratory in terms of its intrinsic interest and conceptual simplicity is Sternberg's (1966, 1969) "item recognition task,". In one version of this experimental paradigm, the "fixed-set procedure," $S$ is given a subspan memory set of stimuli. The $S$ is then presented a series of probe stimuli on discrete trials. Following each probe stimulus, $\mathrm{S}$ is to respond "yes" if the probe is a member of the memory (or "positive") set and "no" if the probe is not a member. The S usually indicates his "yes" or "no" response with finger keys. Results of the task are simple. Reaction time for correct responses usually increases linearly with the number of stimuli in the positive set (m) (e.g., Sternberg, 1966, 1969; Wattenbarger \& Pachella, 1972; cf. Kristofferson, 1972). The monotonic increase of RT with $\mathrm{m}$ is usually taken as evidence of some type of memory search process (e.g., Atkinson, Herrmann, \& Wescourt, 1974; Briggs \& Swanson, 1969; Murdock, 1971; Sternberg, 1966, 1969; Theios, Smith, Haviland, Traupman, \& Moy, 1972; cf. Baddeley \& Ecob, 1973; Cavanagh, 1973; Corballis, Kirby, \& Miller, 1973).

If a card sorting analog of the fixed-set procedure were to produce data similar to the discrete trials version of the task, then an inexpensive simplified procedure

*This research was supported by a grant from the National Institute of Mental Health (MH 21747) to Richard C. Atkinson. The author is grateful to Professor Atkinson for his comments on an earlier draft of this paper and to Dixie Rhodes for her help in running the study.

Requests for reprints should be sent to Lee $D$. Rothstein, Department of Psychology, Stanford University, Stanford, California 94305. would be available for studying the time of memory retrieval processes. Perhaps, then too, other information processing classification tasks could be adapted to a card sorting procedure.

In the present experiment, I attempted to replicate certain aspects of Sternberg's findings with respect to item recognition. As in the fixed-set procedure, $S$ was first given a subspan memory set of digits. Following the memory set, $\mathrm{S}$ was given a deck of cards. Stamped on each card was one of the digits $0-9$. The $E$ then told $S$ to begin sorting the cards into a "yes" pile if the digit on the card was a member of the memory set, or into a "no" pile otherwise. The dependent variable was average sorting time per card. The principle independent variable, as in the item recognition task, was size of the memory set.

\section{METHOD}

\section{Subjects}

Fifteen Stanford undergraduates served as Ss. The Ss were a mixture of students fulfilling course requirements for introductory psychology and paid volunteers. Volunteers were paid $\$ 2.50$ for the $1-\mathrm{h}$ session.

\section{Stimulus Materials}

The cards employed in the task were halves of standard $3 \times 5$ in. index cards. The dimensions of the finished cards were 3 in. high $\times 2.5 \mathrm{in}$. wide. Imprinted on the face of each card in the approximate center was one of the digits $0-9$. The digits were approximately $2.5 \mathrm{~cm}$ high $\times 1.6 \mathrm{~cm}$ wide. The digits were printed using hand-held rubber stamps. The digits 6 and 9 were identical in shape but for a line drawn beneath the base of the 6 . Because the cards were somewhat difficult to separate, $S$ sorted with his fingers coated with paper sorting gel.

Each deck that $\mathrm{S}$ sorted consisted of 60 cards. Memory sets were selected randomly within the constraint that all digits occurred equally often within memory sets of any given set size. Decks were composed so that half of all digits within a deck were members of the positive set, while the other half were members of the "negative" set (the complement to the positive set within the ensemble 0-9). Within the positive and negative sets, stimuli were selected, as much as was possible, equally often. These constraints are the same as the usual constraints of item recognition, namely, that "yes" and "no" responses be equiprobable and that all negative set stimuli occur equally often and all positive set stimuli occur equally often (cf. Sternberg, 1966). 


\section{Procedure}

The $\mathrm{S}$ was seated at a table. Down the center of the table and perpendicular to $S$ was a red tape line. The $S$ was first given the memory set. The deck of cards was placed in S's nonpreferred hand with the cards face down and the tops of the digits all nearest S's body. The S was told to turn the cards over, one at a time, with the preferred hand, so that the digit was upright when viewed by him. He was told to respond "yes" to the question of the presence of each digit in the previously defined memory set by placing the card to the right of the red line. He was told to respond "no" by placing the card to the left of the red line. The cards were sorted from a face-down position in order to prevent the facilitation of preview (Cattell, 1885). The red line was used instead of, say, labeled bins, because in the usual item recognition manipulation $S$ must maintain the response assignment in memory; the red-line manipulation provides the same demand. Ss were told to sort as quickly as they could while attempting to maintain perfect accuracy.

Each S sorted five decks for each of the five Set Sizes 1-5. Each ordinal set of five decks administered to each $\mathrm{S}$ included all five set sizes. The order of the first set of five decks was randomized within the constraint that $\mathrm{m}$ be balanced with resect to ordinal position across Ss. The order of the remaining four sets of five decks for a $\mathrm{S}$ was a Latin-square ordering of the first set.

\section{RESULTS AND DISCUSSION}

For each deck that $\mathrm{S}$ was required to sort, the sort time for the entire deck was divided by the number of cards in the deck (60) in order to yield the mean sort time per card $(\Delta T)$. Of the five decks per set size administered to each $\mathrm{S}$, only the data of the final three

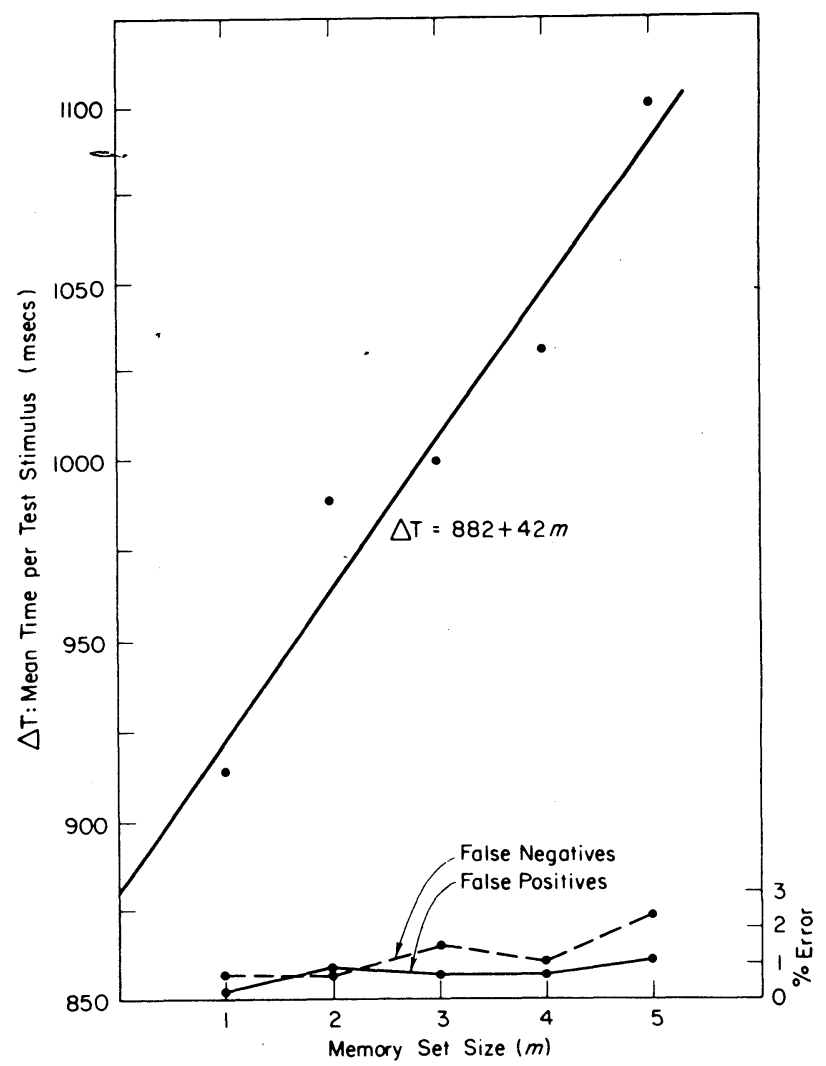

Fig. 1. $\Delta \mathrm{T}$ (mean sorting time per card) as a function of memory set size. were included in the analysis. Figure 1 presents a summary of these results. As can be seen in the figure, $\Delta \mathrm{T}$ is an increasing function of $\mathrm{m}$. The data points do not seem to depart systematically from the best-fit linear function drawn through them. The slope of $42 \mathrm{msec}$ per item is not unlike the slopes I obtain in my laboratory for a discrete trials task, with digits as the stimuli. The $882-\mathrm{msec}$ intercept reflects not only the usual information processing and response components of RT performance, but also motor components of card sorting such as time necessary to turn over a card. Analysis of variance revealed a significant effect of set size on $\Delta T$ $[F(4,56)=43.3, p<.0005]$, consistent with the above description.

At the bottom of Fig. 1 are given the mean percents of errors. Errors occurred very infrequently. The overall error rate was $1.0 \%$. There was a slightly greater tendency toward false negatives than false positives $[1.3 \%$ vs $.7 \%: F(1,14)=4.8, p<.05]$. There was also a slight tendency for errors to increase with $\mathrm{m}$ $[F(4,56)=5.7, p<.001]$. However, this trend was not identical across type of error (false positive vs false negative). The interaction of Error Type by Set Size was significant $[F(4,56)=2.7, p<.05)]$, reflecting an increasing rate of false negatives with increasing $\mathrm{m}$, while false positives remained relatively unaffected by set size. This relationship between error rates is not uncommon, although error rates were uncommonly low.

The experiment demonstrates that a card sorting analog does provide data which in its critical aspects is identical to the discrete trials version of item recognition. The orderliness of the data indicates that valid research on "memory search" can be accomplished using a card sorting procedure. There are, however, some differences in the procedure which require note. First, response type is lost as an independent variable with respect to time. Second, the dependent variable. $\Delta \mathrm{T}$, reflects S's processing time for correct and incorrect responses. In such a procedure the importance of keeping errors to a minimum is critical.

The opportunities for modification of the sorting task to other classification paradigms in order to introduce variety into the undergraduate laboratory seem substantial. The task may be modified to correspond to any "yes"/"no," "true"/"false," or "same"/"different" RT classification task in which it is not critical to distinguish between the two responses. Within the context of psycholinguistics, for example, consider a sentence verification task where number of propositions embedded within a sentence is the independent variable. The two sort piles in this task would be "true" and "false." Each card of a sort deck would have a sentence typed on it. Another possibility would be in the area of semantic memory retrieval. Using a paradigm identical to that of the present experiment and words as the stimuli, one could vary semantic similarity within constant-sized memory sets. Card sorting analogs, then, can give undergraduate psychology students a "taste" of the information processing laboratory. 


\section{REFERENCES}

Atkinson, R. C., Herrmann, D. J., \& Wescourt, K. T. Search processes in recognition memory. In $R$. L. Solso (Ed.) Theories of cognitive psychology: The Loyola symposium. Washington, D.C.: Winston, 1974. Pp. 101-146.

Baddeley, A. D., \& Ecob, J. R. Reaction time and short-term memory: Implications of repetition effects for the high-speed exhaustive scan hypothesis. Quarterly Journal of Experimental Psychology, 1973, 25, 229-240.

Briggs, E. E., \& Swanson, J. M. Encoding, decoding, and central functions in human information processing. Journal of Experimental Psychology, 1970, 86, 296-308.

Cattell, J. M. Uber die Zeit der Erkennung und Benennung-von Schriftzeichen, Bildern und Farben. Philsophische Studien (L eipzig), 1885, 2, 635-650. Translation in A. T. Poffenberger, James McKeen Cattell, Man of Science. Lancaster, Pennsylvania: The Science Press, 1947. Pp. 13-25.

Cavanagh, J. P. Relation between the immediate memory span and the memory search rate. Psychological Review, 1972, 79, 525-530.
Corballis, M. C., Kirby, J., \& Miller, A. Access to elements of a memorized list. Journal of Experimental Psychology, 1972, 94, 185-190.

Kristofferson, M. W. When item recognition and visual search functions are similar. Perception \& Psychophysics, 1972, 12, 379-384.

Murdock, B. B., Jr. A parallel-processing model for scanning. Perception \& Psychophysics, 1971, 10, 289-291.

Sternberg, S. High Speed scanning in human memory. Science $1966,153,652-654$.

Sternberg, S. Memory scanning: Mental processes revealed by reaction-time experiments. American Scientist, 1969,4 , 421-457.

Theios, J. Smith, P. G., Haviland, S. E., Traupman, J., \& Moy, M. C. Memory scanning is a serial, self-terminating process. Journal of Experimental Psychology, 1972, 97, 323-336.

Wattenbarger, B. L., \& Pachella, R. G. The effect of memory load on reaction time in character classification. Perception \& Psychophysics, 1972, 12, 100-102.

(Received for publication June 14, 1974.)

Bulletin of the Psychonomic Society

1974, Vol. 4 (2A), 75-77

\title{
Age changes in apparent arm length*
}

\author{
J. A. SCHLATER \\ Clark University, Worcester, Massachu setts 01610 \\ A. HARVEY BAKER \\ Educational Testing Service, Princeton, New Jersey 08540 \\ and \\ S. WAPNER \\ Clark University, Worcester, Massachu setts 01610
}

\begin{abstract}
To assess whether an earlier finding-viz., that overestimation of apparent head size was maximal in early childhood and decreased with increase in age (Wapner, 1959)-is generalizable to other body parts, the present study assessed age change in judged length of the outstretched arm with Ss ranging from 7 through 18 years. All age groups underestimated arm length, with the magnitude of underestimation decreasing with increase in age-a pattern opposite to that found for head size. Although the age changes observed for both judged head size and arm length can be described as reflecting an increase in accuracy, such a formulation in terms of accuracy cannot explain why young children maximally overestimate head size and maximally underestimate arm length. It is suggested that future research should explore the possibility that these observed differences reflect differential organization of the body schema at different levels of development.
\end{abstract}

Compared to the body of information amassed concerning body-part size estimation in adults (Fisher, 1970), relatively little research in this area has focused

*This investigation was supported in part by the United Stated Public Health Service Grant MH 00348 from the National Institute of Mental Health. We wish to thank the Leicester, Massachusetts public school system for their cooperation and Irene W. Kostin and Laraine Schwartz for their help at various stages in this research. on children or on age changes. Among the studies using children, several have focused on personality correlates of the body-part size judgments (e.g., Rosenshield, 1962; Shaffer, 1964) rather than on age changes in body-part size judgments per se. One study which examined age changes (Gellert \& Stern, 1964) reported that, with increase in age, judgments of self height increased in precision (i.e., the variability of judgments, as measured 